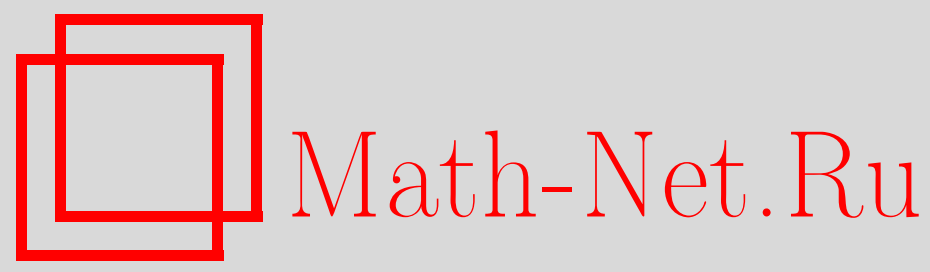

Т. С. Хачлаев, О решении одного нелинейного уравнения второго порядка во внешности компакта, Матем. заметки, 2004, том 76, выпуск 6, 918-921

DOI: https://doi.org/10.4213/mzm155

Использование Общероссийского математического портала Math-Net.Ru подразумевает, что вы прочитали и согласны с пользовательским соглашением http://www . mathnet.ru/rus/agreement

Параметры загрузки:

IP: 18.207 .199 .55

26 апреля 2023 г., $17: 25: 18$

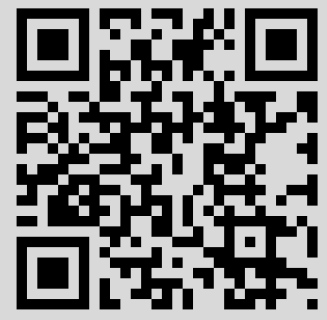




\section{О РЕШЕНИИ ОДНОГО НЕЛИНЕЙНОГО УРАВНЕНИЯ ВТОРОГО ПОРЯДКА ВО ВНЕШНОСТИ КОМПАКТА}

\section{Т. С. Хачлаев}

В работе изучается поведение решений полулинейного эллиптического уравнения в области, являющейся внешностью компакта, при $|x| \rightarrow \infty$. Такого рода уравнения изучались многими авторами (например, Кондратьев, Ландис, Олейник, Верон и др.). В настоящей работе рассмотрен случай, когда в уравнение входят младшие члены. Коэффициенты при младших членах являются произвольными ограниченными измеримыми функциями. Показано, что решения уравнения стремятся к нулю при $|x| \rightarrow \infty$.

Библиография: 2 названия.

Целью настоящей работы является изучение поведения решений полулинейного эллиптического уравнения во внешности компакта.

Рассмотрим уравнение

$$
\sum_{i, j=1}^{n} \frac{\partial}{\partial x_{i}}\left(a_{i j}(x) \frac{\partial u}{\partial x_{j}}\right)+\sum_{i=1}^{n} a_{i}(x) \frac{\partial u}{\partial x_{i}}-a(x)|u|^{\sigma-1} u=0, \quad x \in \Omega,
$$

где $\sigma=$ const $>1, \Omega=\mathbb{R}^{n} \backslash K, K$ - компактное подмножество $\mathbb{R}^{n}, a_{i j}(x), a_{i}(x), a(x)$ ограниченные измеримые функции, $a(x) \geqslant a_{0}=$ const $>0$. Функции $a_{i j}(x)$ удовлетворяют условию равномерной әллиптичности: существуют такие постоянные $\lambda_{1}>0$, $\lambda_{2}>0$, что

$$
\lambda_{1}|\xi|^{2} \leqslant \sum_{i, j=1}^{n} a_{i j}(x) \xi_{i} \xi_{j} \leqslant \lambda_{2}|\xi|^{2} \quad \text { для любых } x \in \Omega, \quad \xi \in \mathbb{R}^{n} .
$$

В качестве решения уравнения (1) понимается обобщенное решение. Приведем его определение. Функция $u(x) \in W_{2}^{1}$ loc $(\Omega) \cap L_{\infty}$ lос $(\Omega)$ назьвается обобщенным решением уравнения (1), если

$$
-\int_{\Omega} \sum_{i, j=1}^{n} a_{i j}(x) \frac{\partial u}{\partial x_{j}} \frac{\partial \Phi}{\partial x_{i}} d x+\int_{\Omega} \sum_{i=1}^{n} a_{i}(x) \frac{\partial u}{\partial x_{i}} \Phi d x-\int_{\Omega} a(x)|u|^{\sigma-1} u \Phi d x=0
$$

Работа выполнена при финансовой поддержке фонда INTAS, грант № 03-51-5007, программы "Ведущие научные школы", грант № НШ-1464.2003.1, и Российского фонда фундаментальных исследований, грант № 02-01-00069. 
для любой $\Phi \in W_{2}^{1}(\Omega)$, имеющей компактньй носитель в $\Omega$.

Основным результатом данной работы является доказательство стремления к нулю всех решений уравнения (1). Для этого потребуется несколько вспомогательных утверждений.

Рассмотрим уравнение (1) в шаре $B_{1}^{0}=\{x:|x|<1\}$. Функция $u(x) \in W_{2}^{1}\left(B_{1}^{0}\right) \cap$ $L_{\infty}\left(B_{1}^{0}\right)$ назьвается решением этого уравнения, если для нее выполнено равенство $(2)$ с произвольной функцией $\Phi(x) \in W_{2}^{1}\left(B_{1}^{0}\right)$, где интегрирование ведется по шару $B_{1}^{0}$.

Лемма 1. Пусть $u(x)$ - произвольное решение уравнения (1) в шаре $B_{1}^{0}$. Тогда $|u(0)|<C$, постоянная $C$ зависит от $\sigma, n$, констант әллиптичности, максимума модуля коэффиичентов $a_{i j}(x), a_{i}(x)$ и от $a_{0}$.

ДокАЗАТЕЛЬСТво этой леммы в случае $a_{i}(x) \equiv 0$ имеется в работе [1].

Лемма 2. Пусть $и(x)$ - обобщенное решение уравнения

$$
\sum_{i, j=1}^{n} \frac{\partial}{\partial x_{i}}\left(a_{i j}(x) \frac{\partial u}{\partial x_{j}}\right)+\sum_{i=1}^{n} a_{i}(x) \frac{\partial u}{\partial x_{i}}-a(x) u=0
$$

в шаре $|x|<1, a_{i j}(x)$ удовлетворяют условию әллиптичности, $A \geqslant a(x) \geqslant a_{0}=$ const $>0,\left|a_{i}(x)\right| \leqslant A u$

$$
\left.u(x)\right|_{|x|=1}=1 .
$$

Тогда

$$
u(0) \leqslant \delta,
$$

əде $\delta=\delta\left(\lambda_{1}, \lambda_{2}, a_{0}, A, n\right) u \delta<1$.

ДокАЗАТЕЛЬСТво. Функция $v=1-u$ будет удовлетворять уравнению

$$
\sum_{i, j=1}^{n} \frac{\partial}{\partial x_{i}}\left(a_{i j}(x) \frac{\partial v}{\partial x_{j}}\right)+\sum_{i=1}^{n} a_{i}(x) \frac{\partial v}{\partial x_{i}}=a(x)(v-1)
$$

и краевому условию

$$
\left.v(x)\right|_{|x|=1}=0
$$

Рассмотрим уравнение

$$
\sum_{i, j=1}^{n} \frac{\partial}{\partial x_{i}}\left(a_{i j}(x) \frac{\partial w}{\partial x_{j}}\right)+\sum_{i=1}^{n} a_{i}(x) \frac{\partial w}{\partial x_{i}}=f(x),
$$

где

$$
f(x)=\left\{\begin{array}{rr}
-1, & |x| \leqslant \varepsilon \\
0, & |x|>\varepsilon
\end{array}\right.
$$

и его решение, удовлетворяюшее условию

$$
\left.w(x)\right|_{|x|=1}=0 .
$$

Постоянную $\varepsilon$ определим ниже. По принципу максимума имеем

$$
v \geqslant a_{0} w \quad \text { при }|x| \leqslant 1 \text {. }
$$


Покажем, что

$$
w \geqslant \delta_{1}>0 \quad \text { при }|x| \leqslant \varepsilon,
$$

где $\delta_{1}$ зависит только от $\lambda_{1}, \lambda_{2}, A, n$.

Решение задачи (5), (6) имеет вид

$$
w(x)=\int_{|y| \leqslant 1} G(x, y) f(y) d y=-\int_{|y| \leqslant \varepsilon} G(x, y) d y
$$

где $G(x, y)$ - функция Грина задачи Дирихле для уравнения (5) в единичном шаре.

Известно, что

$$
\begin{aligned}
& G(x, y)=0 \quad \text { при }|x|=1, \\
& G(x, y)<0 \quad \text { при }|x|<1, \quad|y|<1, \\
& -G(x, y)=\Gamma(x, y)-g(x, y),
\end{aligned}
$$

где $\Gamma(x, y)$ - фундаментальное решение уравнения $(5), g(x, y)$ - решение уравнения $(5)$ по $x$.

Для функции $\Gamma(x, y)$ вьполнены следующие оценки [2]:

$$
c_{2}|x-y|^{2-n} \leqslant \Gamma(x, y) \leqslant c_{1}|x-y|^{2-n}, \quad x, y \in \mathbb{R}^{n} .
$$

Здесь $n>2, c_{1}, c_{2}=$ const $>0, c_{1}, c_{2}$ зависят только от $\lambda_{1}, \lambda_{2}, A, n$.

Если $|x|<\varepsilon,|y|<\varepsilon$, то из (8) следует, что

$$
\Gamma(x, y) \geqslant \frac{c}{2 \varepsilon^{n-2}} .
$$

Заметим, что согласно (7)

$$
g(x, y)=\Gamma(x, y) \quad \text { при }|x|=1 .
$$

Следовательно, по принципу максимума имеем

$$
\max _{|x| \leqslant 1} g(x, y) \leqslant \max _{|x|=1} \Gamma(x, y) \leqslant \max _{|x|=1} \frac{c_{1}}{|x-y|^{n-2}} .
$$

Если $|y|<1 / 2$, то отсюда получим

$$
\max _{|x| \leqslant 1} g(x, y)<2^{n-2} c_{1} .
$$

В частности, при $|x|<\varepsilon,|y|<\varepsilon, \varepsilon<1 / 2$ имеем

$$
-G(x, y)>\frac{c}{2 \varepsilon^{n-2}}-2^{n-2} c_{1}>2 c
$$

если $\varepsilon$ достаточно мало, т.е.

$$
G(x, y)<-2 c=-c_{3} \quad \text { при }|x|<\varepsilon, \quad|y|<\varepsilon .
$$

Следовательно, при $|x|<\varepsilon$

$$
w(x)=\int_{|y|<1} G(x, y) f(y) d y=\int_{|y| \leqslant \varepsilon}-G(x, y) d y>c_{3} \int_{|y| \leqslant \varepsilon} d y=\delta_{1}>0 .
$$

В частности, $w(0)>\delta_{1}$. Поэтому $v(0) \geqslant a_{0} w(0) \geqslant a_{0} \delta_{1}=\delta_{2}$. Отсюда $v(0)=1-u(0)$ $\geqslant \delta_{2}$. Таким образом, получаем

$$
u(0) \leqslant 1-\delta_{2} \equiv \delta, \quad \delta<1,
$$

что и требовалось доказать. Лемма 2 доказана при $n>2$. 
Если $n=2$, то следует продолжить коэффициенты уравнения и его решение в трехмерное пространство, считая их не зависящими от $x_{3}$, и применить полученное утверждение при $n>2$.

Теорема. Пусть $u(x)$ - решение уравнения (1) в области $\Omega=\mathbb{R}^{n} \backslash K, K$ - компактное подмножсество $\mathbb{R}^{n}, a_{i j}(x), a_{i}(x), a(x)$ - ограниченные измеримые функиии, $a(x) \geqslant a_{0}=$ const $>0$. Тогда $u(x)$ стремится к нулю при $|x| \rightarrow+\infty$.

ДоКАЗАТЕЛЬСТВо. Предположим, что $u(x)$ не стремится к нулю. Пусть

$$
\varlimsup_{|x| \rightarrow+\infty} u(x)=\gamma
$$

Можно считать $\gamma>0$ (иначе рассмотрим $-u(x)$ вместо $u(x)$ ). Из леммы 1 следует, что $u(x)$ - ограниченная функция и, значит, $\gamma<+\infty$.

Пусть последовательность $\left\{x^{m}\right\}$ такая, что $\left|x^{m}\right| \rightarrow \infty, u\left(x^{m}\right) \rightarrow \gamma$. В силу гёльдеровости решения $u(x)$ сушествует последовательность шаров одинакового радиуса с центрами в точках $x^{m}$ такая, что

$$
\gamma-\varepsilon<u(x)<\gamma+\varepsilon
$$

в этих шарах. Функция $u(x)$ является решением линейного уравнения

$$
\sum_{i, j=1}^{n} \frac{\partial}{\partial x_{i}}\left(a_{i j}(x) \frac{\partial u}{\partial x_{j}}\right)+\sum_{i=1}^{n} a_{i}(x) \frac{\partial u}{\partial x_{i}}+q(x) u=0
$$

где $q(x)=a(x)|u|^{\sigma-1}$. В силу (10) функция $q(x)$ ограничена и $q(x)>a_{0}(\gamma-\varepsilon)^{\sigma-1}=b_{0}$. По лемме $2 u\left(x^{m}\right)<\delta(\gamma+\varepsilon), \delta<1$, или, переходя к пределу по $m, \gamma<\delta(\gamma+\varepsilon)$. Полученное противоречие доказьвает теорему.

Автор выражает глубокую благодарность профессору В. А. Кондратьеву, под руководством которого были получены эти результаты.

\section{СПИСОК ЦИТИРОВАННОЙ ЛИТЕРАТУРЫ}

[1] Кондратьев В. А., Л андис Е. М. О качественных свойствах решений одного нелинейного уравнения второго порядка // Матем. сб. 1988. Т. 135. № 3. С. 346-360.

[2] Stampacchia G. Le problème de Dirichlet pour les équations elliptiques du second ordre à coefficients discontinus // Ann. Inst. Fourier (Grenoble). 1965. V. 15. P. 189-258.

Московский государственный университет им. М.В. Ломоносова

Поступило

E-mail: hts@elsite.ru

17.02 .2004 